\title{
Phosphodiesterase activity is regulated by CC2D1A that is implicated in non-syndromic intellectual disability
}

Azza Al-Tawashi ${ }^{1,2^{*}}$ and Chris Gehring ${ }^{2}$

\begin{abstract}
Background: Cyclic adenosine $3^{\prime}, 5^{\prime}$-monophosphate (CAMP) is a key regulator of many cellular processes, including in the neuronal system, and its activity is tuned by Phosphodiesterase (PDE) activation. Further, the CC2D1A protein, consisting of N-Terminal containing four DM14 domains and C-terminal containing C2 domain, was shown to regulate the CAMP-PKA pathway. A human deletion mutation lacking the fourth DM14 and the adjacent C2 domain results in Non Syndromic Intellectual Disability (NSID) also referred to as Non Syndromic Mental Retardation (NSMR).

Findings: Here we demonstrate that in Mouse Embryonic Fibroblasts (MEF) CC2D1A co-localizes with PDE4D in the cytosol before CAMP stimulation and on the periphery after stimulation, and that the movement to the periphery requires the full-length CC2D1A. In CC2D1A mouse mutant cells, the absence of three of the four DM14 domains abolishes migration of the complex to the periphery and causes constitutive phosphorylation of PDE4D Serine 126 $\left(S^{126}\right)$ via the CAMP-dependent protein kinase A (PKA) resulting in PDE4D hyperactivity. Suppressing PDE4D activity with Rolipram in turn restores the down-stream phosphorylation of the "CAMP response element-binding protein" (CREB) that is defective in mouse mutant cells.

Conclusion: Our findings suggest that CC2D1A is a novel regulator of PDE4D. CC2D1A interacts directly with PDE4D regulating its activity and thereby fine-tuning CAMP-dependent downstream signaling. Based on our in vitro evidence we propose a model which links CC2D1A structure and function to CAMP homeostasis thereby affecting CREB phosphorylation. We speculate that CC2D1A and/or PDE4D may be promising targets for therapeutic interventions in many disorders with impaired PDE4D function such as NSID.
\end{abstract}

Keywords: Phosphodiesterase activity, CAMP-PKA pathway, CC2D1A, Non-syndromic intellectual disability

\section{Background}

Cyclic AMP regulates a host of cellular functions that include the activation of cAMP-dependent protein kinase A (PKA) that in turn regulates processes such as transcription, cell growth and differentiation, metabolism and ion channel conductivity $[1,2]$. Therefore, alterations in cellular cAMP homeostasis, regulated by adenylate cyclases (ACs) and phosphodiesterases (PDEs) are likely to profoundly affect these cellular processes [3]. The resting concentration of cAMP inside mammalian cells is about

\footnotetext{
* Correspondence: aaltawashi@gmail.com

${ }^{1}$ Center for Molecular Discovery, Verna and Marrs McLean Department of Biochemistry and Molecular Biology, Baylor College of Medicine, 77030, Houston, TX, USA

${ }^{2}$ Division of Chemical and Life Sciences and Engineering, King Abdullah University of Science and Technology, 23955, Thuwal, Saudi Arabia
}

$10^{-7} \mathrm{M}$ and extracellular signals can cause cAMP levels to change by $>20$ fold in seconds which can diffuse rapidly (130 to $700 \mu \mathrm{m}^{2} \mathrm{sec}^{-1}$ ) [4]. In animal cells, cAMP can exert its effects via PKA that consists of a tetrameric holoenzyme composed by two regulatory subunits constitutively linked to two catalytic subunits [5]. Upon stimulation cAMP binds to the regulatory subunits, causing a conformational change which results in the release of catalytic subunits. Once separated, catalytic subunits become activated and catalyze the phosphorylation of specific serines or threonines of target proteins [6]. Activated PKA catalytic subunits (Cs) translocate to the nucleus to phosphorylate the cyclic AMP response element (CREB) at Serine $133\left(\mathrm{~S}^{133}\right)$ initiating the transcription of target genes [7-10]. Compartmentalization of the cAMP active pool occurs through the cAMP module binding to A

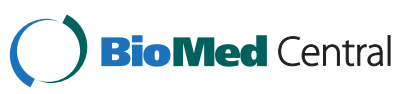


Kinase Anchoring Proteins (AKAPs) [11]. Tethering the PKA regulatory subunit(R) to specific subcellular sites by binding AKAPs helps to assure specific phosphorylation of specific targets and thus prevents uncontrolled phosphorylation. PDEs in turn reduce cAMP concentration thereby tuning the signal down or turning it "off" [12]. A number of distinct isotypes of PDEs modulate the amplitude, length and subcellular distribution of the cAMP signal [13]. Intracellular localization of PDEs also contributes to compartmentalization of cyclic nucleotide signaling [14]. Activation of PKA is tuned by the activity of PDE4D that decreases cAMP levels at the site where PKA is located while PKA phosphorylation of PDE4D is important to fully activate PDE4 enzymes $[15,16]$. This balanced system enables discrete signaling.

Recently, CC2D1A has been shown to be a novel regulator of the cAMP-PKA pathway [17]. Amino acid sequence analyses of the Coiled-coil and C2 domain-containing 1A (CC2D1A) suggested that it consists of a $\mathrm{C} 2$ calciumdependent phospholipid-binding domain, and four conserved Drosophila melanogaster 14 (DM14) domains specific to this protein family with uncharacterized function(s) [18]. Mutant mice with a truncated CC2D1A show defective cAMP-PKA activation and CREB $\left(\mathrm{S}^{133}\right)$ phosphorylation [17].

Interestingly, in NSID patients, the CC2D1A mutant protein has only the first three of the four DM14 domains and carriers have no physical defects but are intellectually disabled [19,20], while the mouse mutant CC2D1A has only a single intact DM14 domain causing death eight to twelve hours after birth, pointing to an essential role of the second and third DM14 domains. Here we set out to characterize the role of CC2D1A during CAMP-dependent stimulation and suggest that its specific function may make a promising drug target.

\section{Results and discussion PDE4D co-localizes with CC2D1A before and after CAMP signaling stimulation} CC2D1A was previously shown to associate with PDE4D5 even in the CC2D1A mutant cells and in brain tissue [17]. In order to characterize CC2D1A interactions with PDE4D5, a series of in vitro pull-down experiments were performed (Figure 1). The different recombinant GST-tagged CC2D1A proteins (fragments I, II, III, and VII) (Figure 1A) were immobilized on glutathione beads and incubated with purified PDE4D5 (IX) (Figure 1A) and PDE4D5-binding was assessed by western blot. PDE4D5 binds to full-length CC2D1A (I) and the CC2D1A (III) fragments, but not to the CC2D1A (VII) fragment suggesting that CC2D1A DM14 domains are essential for binding PDE4D5 (Figure 1B). In addition, CC2D1A-PDE4D5 binding was almost completely abolished in the absence of the first DM14 domain (fragment
II) (Figure 1C). This is consistent with previously reported observations that PDE4D5 can be immunoprecipitated with the mouse CC2D1A mutant form that contains only the first DM14 domain [17], a construct that is similar to fragment VI. We therefore conclude, firstly, that CC2D1A binds PDE4D5 directly and that this binding occurs on the N-terminus and within the DM14 domains and secondly, that the first DM14 domain is essential for the binding. Thirdly, the $\mathrm{C} 2$ domain is not required for binding.

Given that firstly, CC2D1A migrates to the cell periphery after cAMP-stimulation [17] and, in vitro binding of CC2D1A to PDE4D5 (Figure 1), we tested if PDE4D colocalizes with CC2D1A at the periphery. To test this we stimulated wild type (wt) and CC2D1A mutant Mouse Embryonic Fibroblast (MEF) cells with forskolin, fixed them and co-stained them with anti-CC2D1A and antiPDE4D antibodies. The results show that PDE4D and CC2D1A co-localize in the cytosol prior to stimulation and accumulate at the cell periphery after stimulation (Figure 2A). Additionally, although the CC2D1A - PDE4D co-localization in the cytosol was observed in the CC2D1A mutant cells before stimulation, accumulation at periphery does not occur after stimulation indicating the importance of CC2D1A and PDE4D binding in PDE4D accumulation at the periphery (Figure 2A).

\section{The CC2D1A-PDE4D binding regulates PDE4D activity}

Since PKA phosphorylation of PDE4D $\left(S^{126}\right)$ causes activation [21], we investigated whether PDE4D phosphorylation was affected in $C C 2 D 1 A$ mutant MEF cells. When cells were stimulated with forskolin, lysed and western blotting was performed using anti-phospho-PDE4D and anti-PDE4D antibodies, we noted that the level of PDE4D phosphorylation was consistently increased in the mutant $(n=7)$ suggesting that PDE4D may be more active in the mutant even before stimulation which corresponds with CREB phosphorylation defect in the CC2D1A mutant cells on the same western blot (Figure 2B). To validate the sample loading and the phospho-PDE4D and phospho-CREB bands, we re-stained the same blot with anti-PDE4D and anti-CREB (Figure 2C). Given that PDE4 activity increases by $2-3$ fold after PKA has phosphorylated PDE4D and given our observation of PDE4D hyper phosphorylation in CC2D1A mutant cells, we tested if CC2D1A binds PDE4D thereby reducing phosphorylation and activation. The wt and $C C 2 D 1 A$ mutant MEF cells were stimulated with forskolin for different lengths of time, then collected and lysed, protein concentrations were normalized and endogenous PDE4 activity assayed. While PDE4 activity increases and decreases gradually with increasing time of forskolin stimulation in wt cells, PDE4 activity is higher in CC2D1A mutant cells even before stimulation and increases rapidly after the first time point of forskolin stimulation and stays elevated for longer (Figure 2D) indicating 

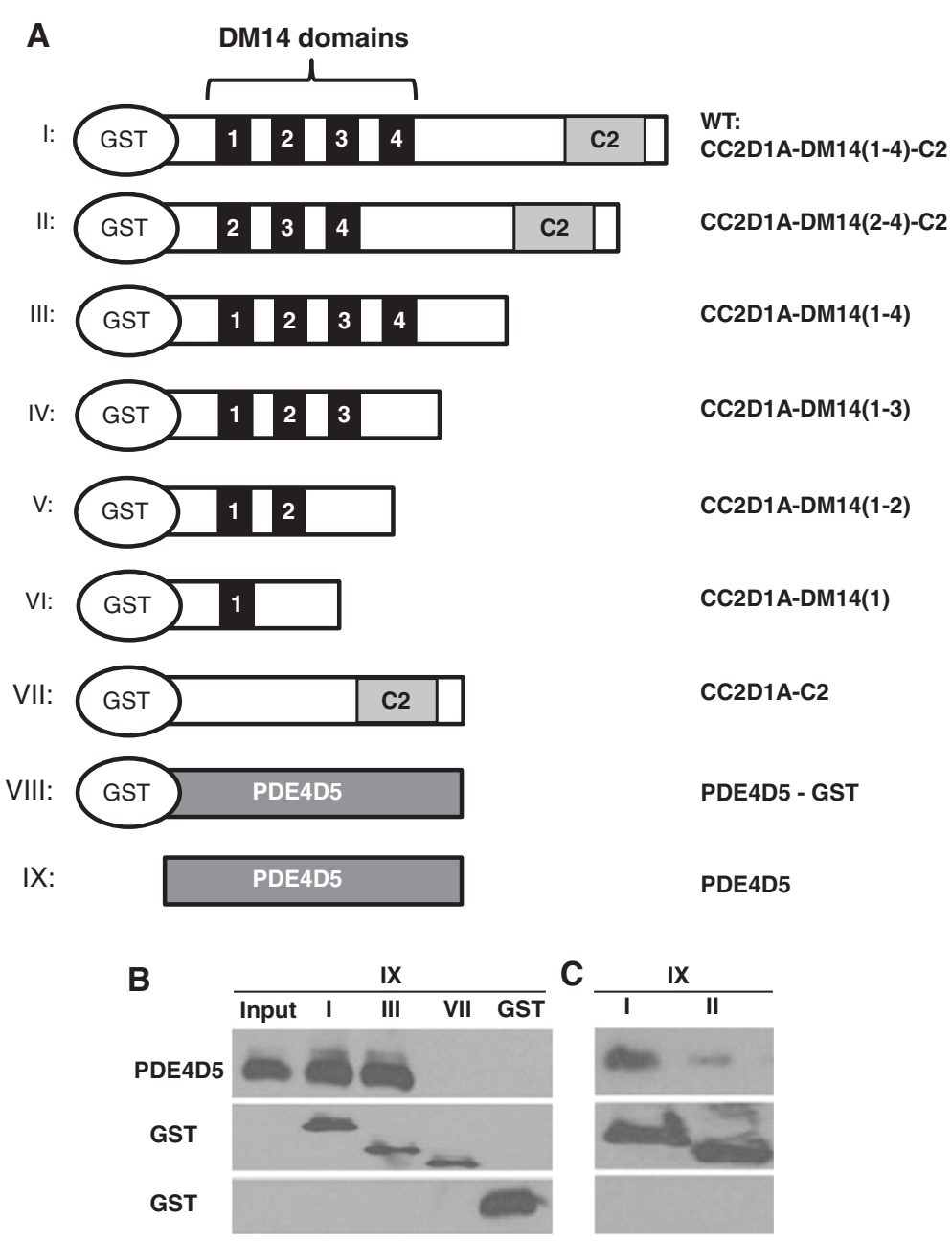

Figure 1 In vitro-binding of CC2D1A to PDE4D5. A. Schematic diagram of GST-CC2D1A and GST-PDE4D5 fusion constructs. B. Western blot of in vitro binding assays of recombinant proteins CC2D1A (fragments I, III, VII and GST) and recombinant PDE4D5 (fragment IX) probed with anti-PDE4D (upper panel) or anti-GST (middle panel and lower panel). The "Input" was purified recombinant PDE4D5 (fragment IX). C. Western blot of in vitro binding assays of recombinant proteins CC2D1A (fragments I and II) and recombinant PDE4D5 (fragment IX) probed with anti-PDE4D (upper panel) or anti-GST (middle panel and lower panel).

that CC2D1A affects PDE4 activity. To test whether this regulation occurs as a result of CC2D1A-PDE4D binding, we first used the PDE4D5 (IX) plasmid and the GSTCC2D1A (I) plasmid (Figure 1) to assay PDE4D5 recombinant activity before and after in vitro phosphorylation by PKA and found that PDE4D5 activity increases approximately two fold after phosphorylation by PKA (Figure 2E) and this is consistent with the previously published data $[21,22]$. Then the effect of CC2D1A-PDE4D binding on PDE4D5 activity in vitro was examined by incubating GST-CC2D1A (I) protein with PDE4D5 (IX) in the presence and absence of PKA. When CC2D1A was bound to PDE4D5 the activity was not affected by PKA suggesting that CC2D1A-binding PDE4D may prevent activation by PKA phosphorylation (Figure 2E). This is supported by the fact that PDE4D5 activity increased after incubation with PKA and prior to the addition of CC2D1A (I)
(Figure 2E). To further investigate if this regulation acts by preventing the PDE4D phosphorylation by PKA, we incubated GST- CC2D1A (I) with PDE4D5 (IX) for in vitro binding, added PKA for in vitro phosphorylation and western blot to examine PDE4D5 phosphorylation at $\left(S^{126}\right)$. The results show that PDE4D5 phosphorylation is dramatically reduced after binding to full-length CC2D1A (I) (Figure 3A) while PDE4D5 phosphorylation increased after incubation with PKA and prior to the addition of CC2D1A (I) (Figure 3A and B). PDE4D5 (IX) activation by PKA was assayed after interaction with different CC2D1A fragments (Figure 1A) in vivo to determine which DM14 domains are critical for PDE4D activity. PDE4D5 (IX) displayed normal activity before and after activation by PKA (Figure 3C) and could be inhibited by the PKA inhibitor (PKI) indicating that the activity is a consequence of the activation by PKA (Figure $3 \mathrm{C}$ ). In agreement 

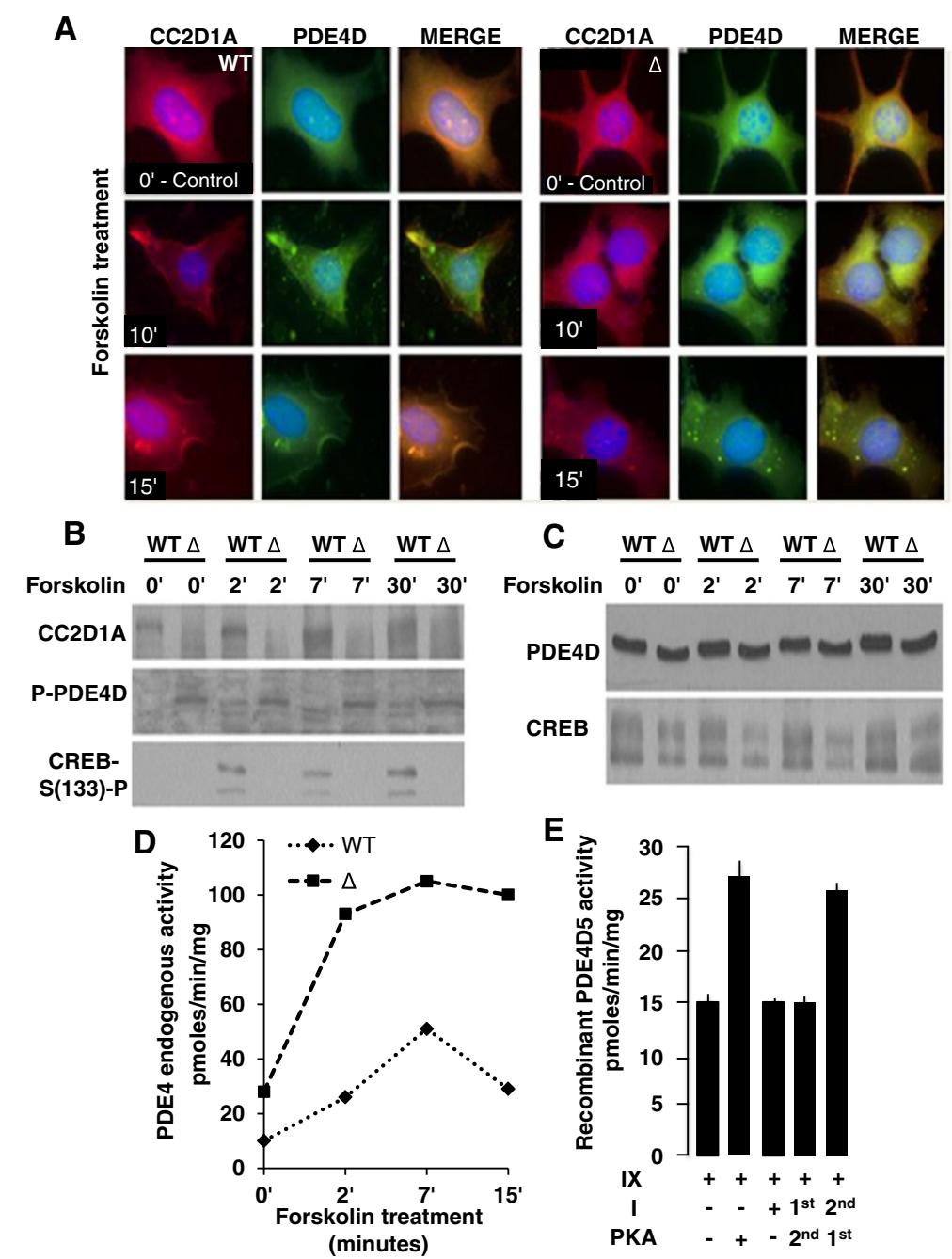

C WT $\Delta$ WT $\Delta$ WT $\Delta$ WT $\Delta$
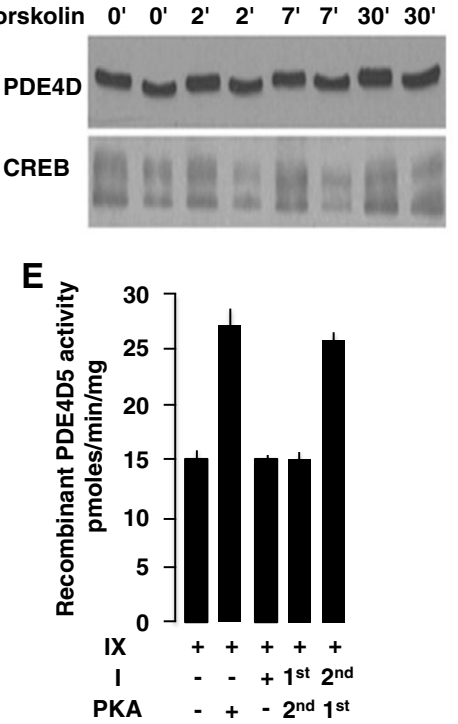

Figure 2 CC2D1A regulates PDE4D activity. A. Immunocytochemistry of forskolin induction time course 0, 10 and 15 minutes of wt and CC2D1A mutant $(\Delta)$ Mouse embryonic fibroblasts (MEF) co-stained with anti-CC2D1A and anti-PDE4D. The mouse mutant form $(\Delta)$ is similar to fragment VI since it contains only the first DM14 domain. B. Western blot of forskolin induction time course 0, 2, 7 and 30 minutes of wt and CC2D1A mutant $(\Delta)$ MEF cells probed with anti-CC2D1A (upper panel), anti- phospho PDE4D (middle panel) or anti-phospho CREB (lower panel) $(n=7)$. C. Western blot of forskolin induction time course $0,2,7$ and 30 minutes of wt and CC2D1A mutant $(\Delta)$ MEF (shown in $\mathbf{B})$ cells probed with anti-PDE4D (upper panel) or anti-CREB (lower panel). D. Endogenous PDE4 activity in forskolin stimulated wt and CC2D1A mutant ( $\triangle$ ) MEF cells. E. In vitro recombinant basal PDE4D5 (fragment IX) activity in the presence and absence of PKA and/or CC2D1A (I).

with the PDE4D5 phosphorylation results (Figure 3A), PKA does not appear to effect PDE4D5 activity after preincubation with the full length CC2D1A (I) and CC2D1A (III and IV) fragments separately (Figure 3C). Although in vitro binding results confirm that the first DM14 domain (Figure 1C) is essential for CC2D1A-PDE4D binding, the results from (Figure 3C) suggest that fragment VI, cannot prevent the increase in PDE4D5 activity after PKA-dependent phosphorylation. The results therefore suggest that the first three DM14 domains (fragment IV) are required to substantially reduce of PDE4D5 activity. Based on that, we conclude that the first three DM14 domains are required to reach near wt regulation of PDE4D5 activity (Figure 3C). The CC2D1A-C2 (VII) fragment does not prevent the increase in PDE4D5 activity after PKA-dependent phosphorylation (Figure 3C).

Rolipram, a PDE4 specific inhibitor restores the CREB S133 $\left(S^{133}\right)$ phosphorylation in CC2D1A mutant cells

Since PDE4 is more active in CC2D1A mutant cells, we hypothesized that suppressing PDE4 activity may compensate for the defective phosphorylation of the PKA target CREB at $\left(\mathrm{S}^{133}\right)$ in CC2D1A mutant cells [17]. To test this we treated wt and CC2D1A mutant MEF cells with a PDE4 specific inhibitor Rolipram [23] prior to stimulation with forskolin, monitored subsequent CREB phosphorylation at $\left(\mathrm{S}^{133}\right)$. Intriguingly, the results indicated that CREB $\left(\mathrm{S}^{133}\right)$ phosphorylation in the CC2D1A 


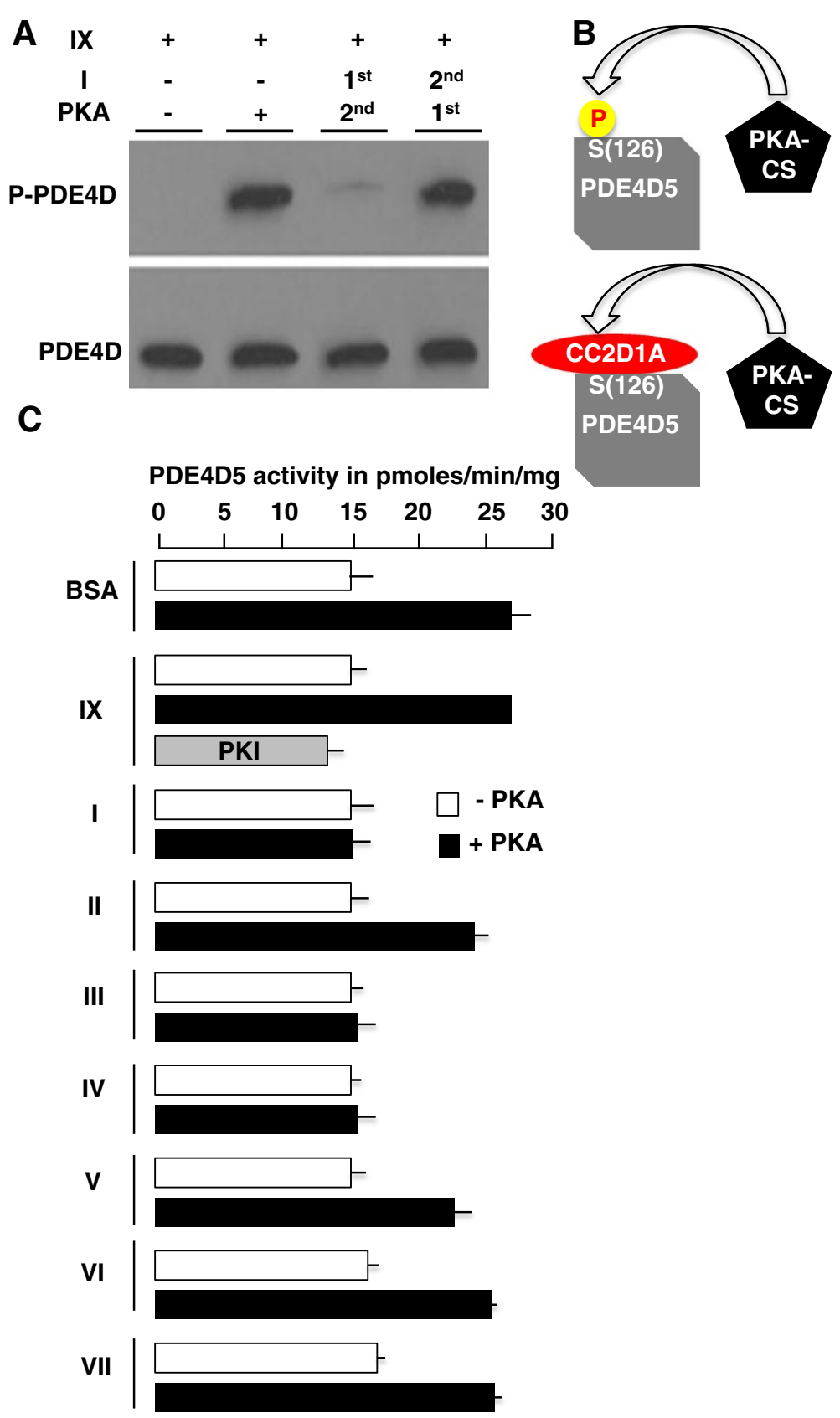

Figure 3 Mapping of the function of DM14 domains. A. Western blot of recombinant PDE4D5 protein (fragment IX) probed with antiphospho PDE4D or anti-PDE4D in the presence and absence of PKA or CC2D1A (I). B schematic diagram of PDE4D phosphorylation at (S ${ }^{126}$ ) by PKA in the presence and absence of CC2D1A. C. In vitro PDE4D activity assay of recombinant PDE4D5 (IX) after pre-incubation with BSA, PKI (specific PKA inhibitor) or CC2D1A (fragments I, II, III, IV, V, VI, VII) in the presence and absence of PKA. BSA was used as a control to validate the specificity of the responses of the different CC2D1A constructs on the inhibition of PDE4D5 activity in the presence of PKA.

mutant cells was restored to wt levels suggesting that PDE4-hyper activity in the mutant might be lowering the cAMP levels leading to defective PKA activity and thereby defective CREB $\left(\mathrm{S}^{133}\right)$ phosphorylation (Figure $\left.4 \mathrm{~A}\right)$.

The cAMP-dependent signaling is essential for many cellular processes including cellular homeostasis and development $[24,25]$. Consequently, spatial and temporal regulation of cellular cAMP concentrations needs to be maintained under tight control. This control is largely exerted by PDEs [26] and more recently, CC2D1A has also been implicated in the control of cAMP homeostasis [17]. The CC2D1A protein contains four DM14 domains 
A
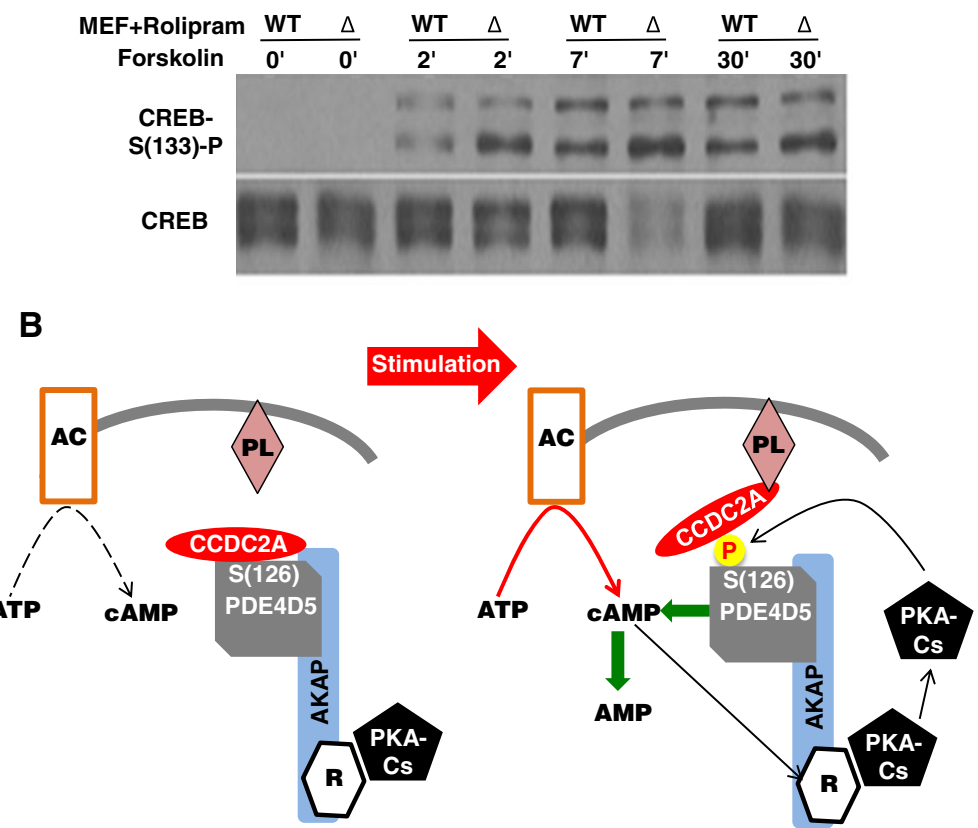

Figure 4 Model of CC2D1A-PDE4D interactions. A. Western blot of time course forskolin induction (0, 2, 7 and 30 minutes) of wt and CC2D1A mutant $(\Delta)$ MEF cells after pre incubation with Rolipram, a specific PDE4 inhibitor, probed with anti-phospho CREB $\left({ }^{133}\right)$ or anti-CREB. B. Schematic model of the mechanism by which CC2D1A regulates the negative feedback mediated by PDE4D.

(Figure 1A) that in turn are annotated only on the basis of amino acid sequence comparisons, but their biochemical and cellular functions have remained elusive [20,27].

While essential and sufficient for binding, the first DM14 of CC2D1A is not sufficient to confer wt function. Humans lacking the fourth DM14 domain of CC2D1A are intellectually disabled but have no other discernible symptoms suggesting that CC2D1A-dependent regulation is particularly critical in developing neural tissue. In turn, the mouse with a CC2D1A mutation that lacks all but the first DM14 has an even more severe phenotype causing death shortly after birth [17]. The results suggest that the absence of DM14 domains 2 and 3 leads to PDE4D hyperphosphorylation at $\left(\mathrm{S}^{126}\right)$, a reaction that is catalyzed by PKA. This hyper-phosphorylation leaves PDE4D constitutively activated and consequently disturbs cAMP homeostasis and cAMP-dependent downstream processes and notably CREB phosphorylation at $\left(\mathrm{S}^{133}\right)$. The latter can be restored by the PDE4 inhibitor Rolipram suggesting that suppressing PDE4D activity may alleviate the effects of the defective phosphorylation of the PKA target CREB at $\left(\mathrm{S}^{133}\right)$ in CC2D1A mutant cells. If CREB phosphorylation is disturbed, it is likely to lead to neural defects and abnormal brain development causing impairments in mental function [10]. The fact that Rolipram has therapeutic benefits as an antidepressant and as an antipsychoticum [28] is further indirect evidence that PDE4D may play a key role in the nervous system and it is noteworthy that disturbances in intracellular cAMP levels and PKAdependent CREB phosphorylation have recently been reported to cause defects in neural crest lineages which in turn manifest themselves as Familial Dysautonomia (FD) syndrome [29]. Given that the mutant CC2D1A protein in NSID patients has the first three DM14 domains intact but is lacking the fourth, we believe that the fourth domain also has a role in CC2D1A regulating PDE4D5 and may be causative for the human syndrome. We speculate that CC2D1A binding to phospholipids [17] at the membrane introduces conformational changes exposing the PDE4D5 (S126) allowing its phosphorylation and activation. Indeed, our ongoing research indicates that the fourth DM14 domain assures the correct in vivo CC2D1A configuration prior to binding to the phospholipid. If this configuration is impaired it is likely to affect PDE4D5 regulation in vivo, and with it cellular cAMP homeostasis. However, the biological role and molecular mechanism of the fourth DM14 domain awaits further testing in vivo.

Here we propose a model (Figure 4B) that links spatial observations to structural and functional aspects of cAMP-dependent phosphorylation. Spatial association of CC2D1A with PDE4D both in the cytosol and, after cAMP stimulation, at the periphery suggests that the common localization may be part of cAMP homeostasis and the regulation of cAMP-dependent processes. In the proposed model for PDE4D5 regulation, upon activation of the adenylate cyclase, cAMP levels increase and cAMP- 
dependent signaling occurs. The CC2D1A-PDE4D-PKA complex relocates to the plasma membrane along a cAMP gradient where CC2D1A will prevent the early PDE4D phosphorylation and activation by PKA. At the membrane, CC2D1A anchors the complex to the cell membrane by binding phospholipids and modulate PKA activity by keeping PDE4D5 inactive for longer allowing a longer signal duration (Figure 4B). Binding of the $\mathrm{C} 2$ domain of the CC2D1A to a membrane phospholipid [17] might cause conformational changes in CC2D1A exposing the $\left(\mathrm{S}^{126}\right)$ residue of PDE4D5 that in turn will be activated by the catalytic subunit of PKA that is released after cAMP activation (Figure 4B). Binding of CC2D1A to the phospholipid un-protects $\left(S^{126}\right)$, allowing PDE4D5 activation that then reduces cAMP levels and turns the signal "off". We propose that CC2D1A regulates the cAMP/PKA pathway through fine-tuning the negative feedback loop that acts via PKA activation of PDE4D. When this pathway is defective, e.g. during neuronal development, it may lead to a state of compromised dendrite growth, synapse formation and neuronal survival [17] and this in turn may be a cause of NSID.

Given that constitutive PDE4D phosphorylation at the PKA site leads to higher enzymatic activity in CC2D1A mutant cells, we propose that $C C 2 D 1 A$ has a role in preventing early PDE4D (hyper-) phosphorylation and activation which in turn would enable higher amplitude of cAMP and longer signal duration to exert the full function effects. Our finding revealed the uncharac terized function of the DM14 domain that, if proven in vivo, may be used in the future as a target for novel therapeutic agents in treatment of diseases caused by PDE4D5-linked disorders.

\section{Conclusion}

The findings presented here suggest that CC2D1A with its DM14 domains is a novel regulator of PDE4D and that specific and spatially correct binding of CC2D1A to PDE4D regulates the activity of the phosphodiesterase that is necessary for fine-tuning cAMP-dependent downstream signaling. We speculate that CC2D1A may be a promising target for therapeutic interventions in conditions with impaired PDE4D5 function such as NSID.

\section{Material and methods}

\section{Glutathione S-transferase (GST)-CC2D1A fusion protein}

A BamHI-EcoRI cDNA fragments encoding the proteins and protein fragments (see Figure 1) cloned into the vector pGEX-4T1 using CC2D1A cDNA in a V5 vector as template. The PCR product and pGEX-4T were digested with BamHI and EcoRI, gel purified, and ligated over night at $16^{\circ} \mathrm{C}$. Ligated plasmids were transformed into E. coli $\mathrm{DH} 5 \alpha$ and plated on agarose gel plates with Ampicillin for selection. For GST-fusion protein purification, overnight cultures of the pGEX-CC2D1A strains were grown in Luria-Bertani broth (LB) with 50 $\mu \mathrm{g} / \mathrm{ml}$ of Ampicillin to an optical density of 0.45 at 600 $\mathrm{nm}$. Isopropyl $\beta$-D-1-thiogalactopyranoside (IPTG) was added to a final concentration of $0.4 \mathrm{mM}$, and the mixture was placed in a shaker at $37^{\circ} \mathrm{C}$ for $4 \mathrm{~h}$. Cells were pelleted and resuspended in $5 \mathrm{ml}$ of phosphate-buffered saline (PBS) plus protease inhibitors $(0.5 \mathrm{mM}$ phenylmethylsulfonyl fluoride, $0.8 \mathrm{mg} / \mathrm{ml}$ leupeptin, $0.8 \mathrm{mg} / \mathrm{ml} \mathrm{pep-}$ statin, and $0.1 \mathrm{mM}$ EDTA) and the mixture was left on ice for $10 \mathrm{~min}$. and then sonicated three times for 10 seconds each. Triton X-100 was then added to a final concentration of $0.1 \%$, and the lysate was shaken gently at $4{ }^{\circ} \mathrm{C}$ for $30 \mathrm{~min}$. Cell debris was cleared by centrifugation at 15,000 $\mathrm{rpm}$ at $4^{\circ} \mathrm{C}$ for $30 \mathrm{~min}$., and the supernatant was transferred to a new microcentrifuge tube with $100 \mu \mathrm{l}$ of $5 \%$ slurry glutathione-conjugated beads. The lysate-GST bead slurry was incubated for $1 \mathrm{~h}$ at $4^{\circ} \mathrm{C}$ with gentle rocking. After that, the slurry was centrifuged at $1,000 \mathrm{rpm}$ for $30 \mathrm{~s}$, the supernatant was removed, and the beads were gently resuspended in $500 \mu \mathrm{l} \mathrm{PBS}$. The centrifugationresuspension was repeated three times to wash the beads free of most contaminating lysate proteins. The GST beads were then resuspended in $100 \mu \mathrm{l}$ of PBS for further use. To cleave the GST tag from the proteins biotinylated Thrombin (Novagen) was used. First, $10 \mu \mathrm{l}$ of thrombin was added ( 1 unit $/ \mu \mathrm{l}$ in $50 \mathrm{mM}$ sodium citrate, $\mathrm{pH} 6.5$, $200 \mathrm{mM} \mathrm{NaCl}, 0.1 \%$ Polyethylene glycol (PEG)-8000, 50\% glycerol) to the $200 \mu \mathrm{l}$ of the bead slurry (100 $\mu \mathrm{l}$ beads and $100 \mu \mathrm{l}$ PBS), followed by $20 \mu \mathrm{l}$ of $10 \mathrm{X}$ thrombin cleavage buffer (200 mM Tris- $\mathrm{HCl}$ pH 8.4, $1.5 \mathrm{M} \mathrm{NaCl}, 25 \mathrm{mM}$ $\mathrm{CaCl} 2$ ) and the mixture was gently shaken at room temperature for $8 \mathrm{~h}$. To capture the thrombin, $200 \mu \mathrm{l}$ of streptavidin agarose beads ( $50 \%$ slurry, Novagen) were added to each sample and incubated with gently shaking at room temperature for $30 \mathrm{~min}$. After that, samples were centrifuged at $500 \times \mathrm{g}$ for $3 \mathrm{~min}$. and the supernatant containing the cleaved protein was transferred to a new tube.

\section{Analysis of signaling pathways in cultured cells}

Cells were stimulated with Forskolin (Sigma) for 5, 10, 15 and $30 \mathrm{~min}$., washed in cold PBS and collected. For inhibition of PDE4 within cells, wild-type and CC2D1A mutant MEF cells were treated with $10 \mu \mathrm{M}$ Rolipram for $20 \mathrm{~min}$. then stimulated with $20 \mu \mathrm{M}$ forskolin for 2,7 , and $30 \mathrm{~min}$. Whole cell lysates (15 $\mu \mathrm{g}$ of protein) were separated by SDS-PAGE gel and transferred to nitrocellulose membranes. The membranes were incubated for 1 $\mathrm{h}$ at room temperature, or overnight at $4^{\circ} \mathrm{C}$, in the corresponding primary and secondary antibodies membranes developed using the enhanced chemiluminescence (ECL) detection system (Amersham Biosciences). 


\section{Immunocytochemistry}

Cells were cultured on poly-D-lysine-coated cover slips and after experimental treatments the cells were fixed with $4 \%$ paraformaldehyde in PBS for $10 \mathrm{~min}$. at room temperature, washed 3 times with PBS, incubated for 20 min. at $37^{\circ} \mathrm{C}$ in $\mathrm{PBS}$ containing $5 \%$ goat serum, prior to adding the Anti-PDE4D and Anti-CC2D1A primary antibodies. Following three washes with PBS, the coverslips were incubated for $20 \mathrm{~min}$. at $37^{\circ} \mathrm{C}$ with the Texas Red and Fluorescein isothiocyanate (FITC) conjugated secondary antibody (1/300 dilution, Chemicon). After three washes with PBS the cover slips were incubated for $5 \mathrm{~min}$. on ice in PBS containing $50 \mu \mathrm{g} / \mathrm{ml}$ of 4',6-diamidino2-phenylindole (DAPI), then washed three times with PBS and mounted on glass slides. Antibody signals were visualized with a Delta Vision de-convolution microscope.

\section{In vitro protein binding assay}

We obtained a GST-PDE4D5 plasmid from Graeme B. Bolger (University of Alabama Comprehensive Cancer Center). GST-PDE4D5 (VIII) protein was expressed and purified and cleaved as described above. The four GSTtagged CC2D1A (I, II, III, and IV) proteins that were previously purified on GST beads, and the purified PDE4D5 (IX) protein, were used as follows. The PDE4D5 (IX) $(20 \mu \mathrm{g})$ was added to the $200 \mu \mathrm{l}$ of the GST bead solution with bound GST-CC2D1A (I), GST-CC2D1A (II), GST-CC2D1A (III), or GST-CC2D1A (IV) separately. After incubation at $4^{\circ} \mathrm{C}$ for $4 \mathrm{~h}$, samples were centrifuged at $500 \mathrm{x} \mathrm{g}$ for $1 \mathrm{~min}$. and the supernatant was removed. Washing with PBS and centrifugation were repeated three times and the PBS from the last wash was totally removed. Samples were boiled at $95^{\circ} \mathrm{C}$ with $30 \mu$ of protein loading buffer for $5 \mathrm{~min}$. To assess binding, $20 \mu \mathrm{l}$ of each sample was loaded on an SDS-PAGE gel and duplicate western blots were made and stained with anti-PDE4D affinitypurified rabbit antibody (1/500; FabGennix), or anti-GST mouse monoclonal antibody (GenScript) separately.

\section{PDE4 Assay}

The wt and CC2D1A mutant Mouse Embryonic Fibroblasts (MEF) cells were stimulated with Forskolin, processed and the broken up by sonication. Phosphorylation of the GSTPDE4D5 recombinant protein was assessed after PKA treatment and exposure to ATP (Assay Designs). PDE4 activity was measured using the PDE4 Enzymatic Assay Kit (FabGennix) according to the manufacture's protocol. (The methods applied here are further detailed in the Additional file 1).

\section{Additional file}

Additional file 1: Materials and Methods.

\section{Abbreviations}

ACs: Adenylate cyclases; AKAP: A kinase anchoring protein; CAMP: Cyclic adenosine 3',5'-monophosphate; CC2D1A: Coiled-coil and C2 domaincontaining 1A; CREB: CAMP response element-binding protein;

DM14: Drosophila melanogaster 14 protein; GST: Glutathione S-transferase; MEF: Mouse embryonic fibroblasts; NSID: Non syndromic intellectual disability; PDE: Phosphodiesterase; PKA: CAMP-dependent protein kinase A.

\section{Competing interests}

Both authors declare that they have no competing interests.

\section{Authors' contributions}

AA has designed and performed the experiments; AA and CG have interpreted the data, conceived the model and written the manuscript. Both authors read and approved the final manuscript.

\section{Acknowledgements}

We thank Adam Kuspa, Jun Qin and Sung Yun Jung (Baylor College of Medicine) for valuable discussions and support in this project.

Received: 22 June 2013 Accepted: 26 June 2013

Published: 4 July 2013

\section{References}

1. Antoni FA: Interactions between intracellular free $\mathrm{Ca}^{2+}$ and cyclic AMP in neuroendocrine cells. Cell Calcium 2012, 51:260-266.

2. McDonough KA, Rodriguez A: The myriad roles of cyclic AMP in microbial pathogens: from signal to sword. Nat Rev Microbiol 2012, 10:27-38.

3. de Joussineau C, Sahut-Barnola I, Levy I, Saloustros E, Val P, Stratakis CA, Martinez A: The CAMP pathway and the control of adrenocortical development and growth. Mol Cell Endocrinol 2012, 351:28-36.

4. Grutsch JF, Robertson A: The CAMP signal from Dictyostelium discoideum amoebae. Dev Biol 1978, 66:285-293.

5. Pearce LR, Komander D, Alessi DR: The nuts and bolts of AGC protein kinases. Nat Rev Mol Cell Biol 2010, 11:9-22.

6. Anand GS, Krishnamurthy S, Bishnoi T, Kornev A, Taylor SS, Johnson DA: Cyclic AMP- and (Rp)-cAMPS-induced conformational changes in a complex of the catalytic and regulatory (Rla) subunits of cyclic AMP-dependent protein kinase. Mol Cell Proteomics 2010, 9:2225-2237.

7. Chrivia JC, Kwok RP, Lamb N, Hagiwara M, Montminy MR, Goodman RH: Phosphorylated CREB binds specifically to the nuclear protein CBP. Nature 1993, 365:855-859.

8. Impey S, McCorkle SR, Cha-Molstad H, Dwyer JM, Yochum GS, Boss JM, McWeeney S, Dunn JJ, Mandel G, Goodman RH: Defining the CREB regulon: a genome-wide analysis of transcription factor regulatory regions. Cell 2004, 119:1041-1054.

9. Lee JH, Giannikopoulos P, Duncan SA, Wang J, Johansen CT, Brown JD, Plutzky J, Hegele RA, Glimcher LH, Lee AH: The transcription factor cyclic AMP-responsive element-binding protein $\mathrm{H}$ regulates triglyceride metabolism. Nat Med 2011, 17:812-815.

10. Herold S, Jagasia R, Merz K, Wassmer K, Lie DC: CREB signalling regulates early survival, neuronal gene expression and morphological development in adult subventricular zone neurogenesis. Mol Cell Neurosci 2011, 46:79-88.

11. Skroblin P, Grossmann S, Schafer G, Rosenthal W, Klussmann E: Mechanisms of protein kinase A anchoring. Int Rev Cell Mol Biol 2010, 283:235-330.

12. Burgin $A B$, Magnusson OT, Singh J, Witte $P$, Staker BL, Bjornsson JM, Thorsteinsdottir M, Hrafnsdottir S, Hagen T, Kiselyov AS, et al: Design of phosphodiesterase 4D (PDE4D) allosteric modulators for enhancing cognition with improved safety. Nat Biotechnol 2010, 28:63-70.

13. Brunton LL: PDE4: arrested at the border. Sci STKE 2003, 2003:PE44.

14. Matthiesen K, Nielsen J: Cyclic AMP control measured in two compartments in HEK293 cells: phosphodiesterase K(M) is more important than phosphodiesterase localization. PLoS One 2011, 6:e24392.

15. MacKenzie SJ, Baillie GS, McPhee I, Mackenzie C, Seamons R, McSorley T, Millen J, Beard MB, van Heeke G, Houslay MD: Long PDE4 CAMP specific phosphodiesterases are activated by protein kinase A-mediated phosphorylation of a single serine residue in Upstream Conserved Region 1 (UCR1). Br J Pharmacol 2002, 136:421-433. 
16. Baillie GS, Adams DR, Bhari N, Houslay TM, Vadrevu S, Meng D, Li X, Dunlop A, Milligan G, Bolger GB, et al: Mapping binding sites for the PDE4D5 cAMP-specific phosphodiesterase to the $\mathrm{N}$ - and C-domains of betaarrestin using spot-immobilized peptide arrays. Biochem J 2007 404:71-80.

17. Al-Tawashi A, Jung SY, Liu D, Su B, Qin J: Protein implicated in nonsyndromic mental retardation regulates protein kinase A (PKA) activity. J Biol Chem 2012, 287:14644-14658.

18. Rogaeva A, Albert PR: The mental retardation gene CC2D1A/Freud-1 encodes a long isoform that binds conserved DNA elements to repress gene transcription. Eur J Neurosci 2007, 26:965-974.

19. Basel-Vanagaite L, Attia R, Yahav M, Ferland RJ, Anteki L, Walsh CA, Olender $T$, Straussberg R, Magal N, Taub E, et al: The CC2D1A, a member of a new gene family with $C 2$ domains, is involved in autosomal recessive non-syndromic mental retardation. J Med Genet 2006, 43:203-210.

20. Rogaeva A, Galaraga K, Albert PR: The Freud-1/CC2D1A family: transcriptional regulators implicated in mental retardation. J Neurosci Res 2007, 85:2833-2838.

21. Brown KM, Lee LC, Findlay JE, Day JP, Baillie GS: Cyclic AMP-specific phosphodiesterase, PDE8A1, is activated by protein kinase A-mediated phosphorylation. FEBS Lett 2012, 586:1631-1637.

22. Vandamme J, Castermans D, Thevelein JM: Molecular mechanisms of feedback inhibition of protein kinase A on intracellular cAMP accumulation. Cell Signal 2012, 24:1610-1618.

23. Zanotti-Fregonara P, Zoghbi SS, Liow JS, Luong E, Boellaard R, Gladding RL, Pike WW, Innis RB, Fujita M: Kinetic analysis in human brain of [11C] (R)-rolipram, a positron emission tomographic radioligand to image phosphodiesterase 4: a retest study and use of an image-derived input function. Neuroimage 2011, 54:1903-1909.

24. Abel S, Chien P, Wassmann P, Schirmer T, Kaever V, Laub MT, Baker TA Jenal U: Regulatory cohesion of cell cycle and cell differentiation through interlinked phosphorylation and second messenger networks. Mol Cell 2011, 43:550-560.

25. Antoni FA: New paradigms in CAMP signalling. Mol Cell Endocrinol 2012, 353:3-9.

26. Conti M, Beavo J: Biochemistry and physiology of cyclic nucleotide phosphodiesterases: essential components in cyclic nucleotide signaling. Annu Rev Biochem 2007, 76:481-511.

27. Albert PR, Le Francois B, Millar AM: Transcriptional dysregulation of 5-HT1A autoreceptors in mental illness. Mol Brain 2011, 4:21.

28. Park SJ, Ahmad F, Philp A, Baar K, Williams T, Luo H, Ke H, Rehmann H, Taussig R, Brown AL, et al: Resveratrol ameliorates aging-related metabolic phenotypes by inhibiting cAMP phosphodiesterases. Cell 2012, 148:421-433.

29. Lee G, Ramirez CN, Kim H, Zeltner N, Liu B, Radu C, Bhinder B, Kim YJ, Choi IY, Mukherjee-Clavin B, et al: Large-scale screening using familial dysautonomia induced pluripotent stem cells identifies compounds that rescue IKBKAP expression. Nature Biotechnology 2012, 30:1244-1248.

doi:10.1186/1478-811X-11-47

Cite this article as: Al-Tawashi and Gehring: Phosphodiesterase activity is regulated by CC2D1A that is implicated in non-syndromic intellectual disability. Cell Communication and Signaling 2013 11:47.

\section{Submit your next manuscript to BioMed Central and take full advantage of:}

- Convenient online submission

- Thorough peer review

- No space constraints or color figure charges

- Immediate publication on acceptance

- Inclusion in PubMed, CAS, Scopus and Google Scholar

- Research which is freely available for redistribution

Submit your manuscript at www.biomedcentral.com/submit
C Biomed Central 